\title{
Fluctuations of temperature gradients in turbulent thermal convection
}

\author{
K.R. Sreenivasan ${ }^{1}$, A. Bershadskii ${ }^{1,2}$ and J.J. Niemela ${ }^{1}$ \\ ${ }^{1}$ International Center for Theoretical Physics, Strada Costiera 11, I-34100 Trieste, Italy \\ ${ }^{2}$ ICAR, P.O. Box 31155, Jerusalem 91000, Israel
}

\begin{abstract}
Broad theoretical arguments are proposed to show, formally, that the magnitude $G$ of the temperature gradients in turbulent thermal convection at high Rayleigh numbers obeys the same advectiondiffusion equation that governs the temperature fluctuation $T$, except that the velocity field in the new equation is substantially smoothed. This smoothed field leads to a -1 scaling of the spectrum of $G$ in the same range of scales for which the spectral exponent of $T$ lies between $-7 / 5$ and $-5 / 3$. This result is confirmed by measurements in a confined container with cryogenic helium gas as the working fluid for Rayleigh number $R a=1.5 \times 10^{11}$. Also confirmed is the logarithmic form of the autocorrelation function of $G$. The anomalous scaling of dissipation-like quantities of $T$ and $G$ are identical in the inertial range, showing that the analogy between the two fields is quite deep.
\end{abstract}

PACS numbers: 47.27.Te; 47.27.Jv

While statistical properties of temperature fluctuations in turbulent Rayleigh-Bénard convection have received considerable experimental and theoretical consideration (see, for instance, 1]-12 and the references therein), corresponding properties of temperature gradients are still unexplored from both theoretical and experimental points of view. In the present paper, we study statistical properties of temperature gradients emphasizing their qualitative and quantitative similarity to those of the temperature fluctuations themselves. Theoretical considerations will be based on an equation to be derived for the magnitude of temperature gradients, and the results will be compared with measurements in turbulent convection in a confined container of circular cross-section. We would like to emphasize crucial difference between spectrum of the temperature gradient and spectrum of absolute value (magnitude) of the temperature gradient. The former one can be readily estimated using the Taylor's hypothesis. Namely, the Fourier transform of the time series of the temperature derivative is simply proportional to omega multiplied by the transform of the temperature time series. If this is the case, there should simply be a factor omega squared between the two spectra. If, however, we consider spectrum of absolute value of the temperature gradient, then there is no straightforward relation between this spectrum and spectrum of the original temperature time series. In the last case one need use of physics, and the spectrum (autocorrelation function) of the absolute value of the temperature gradient can give additional information about the thermal convection process.

The measurement apparatus has unity aspect ratio. The sidewalls of the apparatus are insulated, and the bottom and top walls are maintained at constant temperatures; the bottom wall is held at a slightly higher temperature $\Delta$ than the top wall. The working fluid is cryogenic helium gas. We measure temperature fluctuations at various Rayleigh numbers towards the upper end of this range, in which the convective motion is turbulent, but use the data obtained at the Rayleigh number $R a=1.5 \times 10^{11}$ in the present paper. Time traces of fluctuations are obtained at a distance of $4.4 \mathrm{~cm}$ from the sidewall on the center plane of the apparatus. This position is outside of the boundary layer region for the Rayleigh number considered here. At this Rayleigh number, the mean wind (which is the large-scale circulation within the convection apparatus) is well developed so Taylor's hypothesis can be employed when necessary. More details of the experimental conditions and measurement procedure can be found in Ref. 7].

In thermal convection, the temperature field $T(\mathbf{r}, t)$ is convected by the velocity field $\mathbf{v}(\mathbf{r}, t)$, which itself is generated by density differences set up between the top and bottom walls. We will consider incompressible flow obeying $\nabla \cdot \mathbf{v}=0$ (with unit density for simplicity). The relevant equations under the Boussinesq approximation are

$$
\begin{gathered}
\frac{\partial \mathbf{v}}{\partial t}=-\nabla p-(\mathbf{v} \cdot \nabla) \mathbf{v}+\nu \nabla^{2} \mathbf{v}+\alpha g T \hat{z}, \\
\frac{\partial T}{\partial t}=-(\mathbf{v} \cdot \nabla) T+D \nabla^{2} T
\end{gathered}
$$

Here $p, \nu, D, \alpha, g$ and $\hat{z}$ are, respectively, the pressure, kinematic viscosity, thermal diffusivity, isobaric thermal expansion coefficient, acceleration due to gravity, and the unit vector in the upward direction. Equation (2) is the standard scalar advection-diffusion equation, except that the velocity field is coupled to the temperature field. This 'active' nature of the temperature fluctuations in convection makes their statistical properties different from those of a passive scalar advected by a turbulent velocity with no back reaction. Restricting attention, for simplicity, to Prandtl numbers of the order unity, the experimentally measured spectral density of temperature fluctuations in the inertial (convective) range rolls off at a rate that is closer to -1.4 than to $-5 / 3$, the latter being the case for passive scalars in three-dimensional homogeneous turbulence [1, 2, 4, 5, 7]. 
The equation for temperature gradients $\mathbf{G} \equiv \nabla T$ can be readily derived from (2) as

$$
\frac{\partial G_{i}}{\partial t}=-v_{j} \frac{\partial G_{i}}{\partial x_{j}}-\frac{\partial v_{j}}{\partial x_{i}} G_{j}+D \frac{\partial^{2} G_{i}}{\partial x_{j}^{2}},
$$

with the indices $i$ and $j$ representing the space coordinates, and the summation over repeated indexes is assumed. The magnitude $G$ of the temperature gradient is determined by $\mathbf{G}=G \mathbf{n}$, where $\mathbf{n}$ is the unit vector with its direction along vector $\mathbf{G}$. Multiplying both sides of Eq. (3) by $n_{i}$, making summation over $i$, and taking into account of the fact that $n_{i}^{2}=1$, we obtain

$$
\frac{\partial G}{\partial t}=-(\mathbf{v} \cdot \nabla) G+D \nabla^{2} G-\lambda G,
$$

which is formally similar to Eq. (2) except for the last term in (4). The coefficient $\lambda$ in this term has the form

$$
\lambda=n_{i} n_{j} \frac{\partial v_{i}}{\partial x_{j}}+D\left(\frac{\partial n_{i}}{\partial x_{j}}\right)^{2} .
$$

Let us now search for circumstances under which the last term in Eq. (4) is small in the inertial range. The second term in $\lambda$ is assured to be small because the diffusivity $D$ is small. But the nature of the "stretching" part on the right hand side of Eq. (5) is not apparent without further considerations.

As a further step, let us make the following conditional average of Eq. (4). Fix the magnitude $G$ in the vector field $\mathbf{G}=G \mathbf{n}$ while performing the average over all realizations of the direction vector field $\mathbf{n}$ permitted by equation (3). Let us denote this ensemble average as $\langle\ldots\rangle_{\mathbf{n}}$. From the definition, this averaging procedure does not affect $G$ itself, but modifies the velocity field $\mathbf{v}$, which in turn modifies the coefficient $\lambda$ in Eq. (4). We may write

$$
\frac{\partial G}{\partial t}=-\left(\langle\mathbf{v}\rangle_{\mathbf{n}} \cdot \nabla\right) G+D \nabla^{2} G+\langle\lambda\rangle_{\mathbf{n}} G .
$$

It is worth noting that the solutions of Eq. (3) satisfy Eqs. (4) and (6), but not all possible formal solutions of the Eqs. (4) and (6) satisfy Eq. (3); similarly, not all formal solutions of Eq. (6) satisfy Eq. (4) while all solutions of Eq. (4) do satisfy Eq. (6). In particular, the solutions of Eqs. (4) and (6) are the same only if: (a) the initial conditions for the two equations are the same, and (b) if realizations of $\langle\mathbf{v}\rangle_{\mathbf{n}}$ and of $\langle\lambda\rangle_{\mathbf{n}}$, related to these initial conditions by the conditional averaging procedure, are taken from the applicable solutions of Eq. (4).

It is difficult to guess a priori when $\langle\lambda\rangle_{\mathbf{n}}$ is negligible, because there is no small parameter for the stretching part of $\lambda$. Therefore, let us consider a generic set of conditions, presumably for the inertial (convective) range, which can result in $\left\langle n_{i} n_{j} \partial v_{i} / \partial x_{j}\right\rangle_{\mathbf{n}}=0$. This can be a combination of isotropy, which yields

$$
\left\langle n_{i} n_{j}\right\rangle_{\mathbf{n}}=0 \quad(i \neq j)
$$

and

$$
\left\langle n_{1}^{2}\right\rangle_{\mathbf{n}}=\left\langle n_{2}^{2}\right\rangle_{\mathbf{n}}=\left\langle n_{3}^{2}\right\rangle_{\mathbf{n}}
$$

and directional randomness determined by equation

$$
\left\langle n_{i} n_{j} \varphi\right\rangle_{\mathbf{n}}=\left\langle n_{i} n_{j}\right\rangle_{\mathbf{n}}\langle\varphi\rangle_{\mathbf{n}},
$$

where $\varphi=\partial v_{k} / \partial x_{l}$ for arbitrary $k$ and $l$. One should not mix the conditions (7)-(9) with global isotropy and statistical independence of the velocity and temperature gradients. In particular, (7)-(9) can be satisfied in the inertial range even in the presence of strong global anisotropy and correlation between gradients (see below for more comments).

If we use conditions (7)-(9) in the presence of the incompressibility condition $\partial v_{i} / \partial x_{i}=0$ we obtain

$$
\langle\lambda\rangle_{\mathbf{n}}=-D\left\langle\left(\frac{\partial n_{i}}{\partial x_{j}}\right)^{2}\right\rangle_{\mathbf{n}}
$$

That is, the formal difference between Eq. (2) for $T$ and the conditionally averaged Eq. (6) for $G$ is reduced to the " $\lambda$ " term with the $\lambda$ given by Eq. (10). Equation (6) can then be reduced, in Lagrangian variables, to

$$
\frac{d G}{d t}=\langle\lambda\rangle_{\mathbf{n}} G
$$

with the "multiplicative noise" $\langle\lambda\rangle_{\mathbf{n}}$ given by Eq. (10).

Weak diffusion of Lagrangian "particles" can be described as their wandering around the deterministic trajectories. The introduction of a weak diffusion is equivalent to the introduction of additional averaging in Eq. (11) over random trajectories 13. The small parameter $D$ in (10) and (11) will then determine a slow time in comparison with the time scales in the inertial range and will therefore not affect scaling properties of $G$ in that range.

We should emphasize that the conditional average indicated by $\langle\ldots\rangle_{\mathbf{n}}$ is quite different from the global average indicated by $\langle\ldots\rangle$. Because of this, the quantity $G$ in Eq. (6) remains a fluctuating variable. To eliminate the stretching part from the conditionally averaged coefficient $\langle\lambda\rangle_{\mathbf{n}}$, one does not need to satisfy conditions (7)-(9) for all realizations of the temperature gradient field $\mathbf{G}$, but only for the subset of realizations that gives the main statistical contribution to the spectrum of the magnitude $G$ in the inertial range. Therefore, conditions (7)-(9) could well be violated globally without affecting the main conclusion.

The essential point here is that the conditionally averaged velocity $\langle\mathbf{v}\rangle_{\mathbf{n}}$ is smoothed substantially in comparison with $\mathbf{v}$, while the fluctuation of $G$ itself is still rapid in the diffusion-advection equation (6) (because it remains in tact under the conditional average, by virtue of its definition). Under these typical circumstances, the natural expectation (see, for instance, Ref. [14] and references 


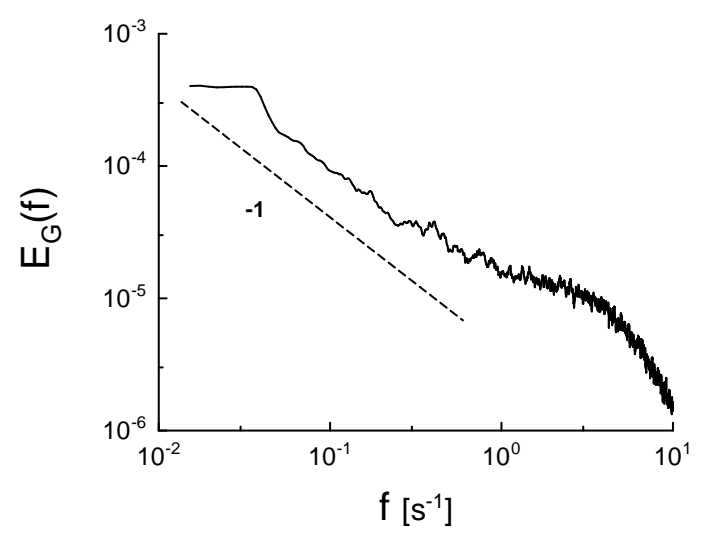

FIG. 1: Spectrum of a one-dimensional surrogate of the magnitude of the temperature fluctuations gradient in thermal convection. The straight line is drawn to indicate the powerlaw spectrum (12).

therein) is that the spectral law for a quantity governed by the diffusion-advection equation has a "-1" region. The result owes itself to the pioneering work of Batchelor [15], who applied this general idea to the viscousconvection range of passive scalar fluctuations. While the two contexts are quite different, they are the same in the sense that the velocity field is smooth.

There is another way of deducing the -1 power law. We recall that the spectral density fluctuations of temperature in this region of scales has an approximately $-7 / 5$-ths slope. This slope can be derived by the dimensional considerations used by Bolgiano [16]. We may apply the same reasoning to Eq. (6). Thus, introducing an analogy of the dissipation rate for $G$, namely $\chi_{*}=d G^{2} / d t$, Bolgiano's dimensional arguments yield the scaling law for $G$ to be

$$
E_{G}(k) \sim\left\langle\chi_{*}\right\rangle^{4 / 5}(\alpha g)^{-2 / 5} k^{-1}
$$

in the inertial range. Figure 1 shows the corresponding spectrum observed for a one-dimensional derivative of $G$ in our experiment. For the effectively smoothed velocity field, the space autocorrelation function can be characterized by a logarithmic behavior [17] given by

$$
C(r)=\langle G(r) G(0)\rangle \sim \ln \left(\frac{L}{r}\right),
$$

or, using Taylor's hypothesis, in terms of $\tau$ by

$$
C(\tau)=\langle G(\tau) G(0)\rangle \sim \ln \left(\frac{\tau_{0}}{\tau}\right) .
$$

This is seen from Fig. 2 to apply quite precisely for the data. In our approach, the active character of the temperature in the convection manifests itself through nontrivial properties of the locally averaged velocity field in

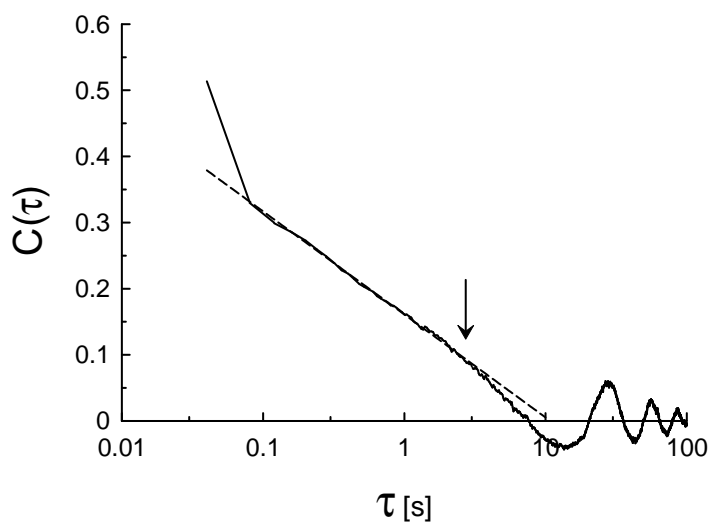

FIG. 2: Autocorrelation function $C(\tau)$ plotted against $\log \tau$, computed for the same data used for the spectral calculations. The vertical arrow here and in Fig. 3 indicates the end of the inertial range.

eq. (6). As a consequence, for instance, the very significant input scale $L$ or $\tau_{0}$ for the autocorrelation function has approximately the same size as typical size of the largest plums observed in the convection. The input scale can be readily calculated from Fig. $2\left(\tau_{0} \simeq 10 \mathrm{sec}\right)$.

The similarity of the $T$ and $G$ fields can also be seen for the scaling of the dissipation rate itself. The local temperature dissipation can be characterized by a gradient measure [16] as

$$
\chi(r)=\frac{\int_{\Omega_{r}}(\nabla T)^{2} d v}{v_{r}}
$$

where $\Omega_{r}$ is a subvolume with space-scale $r$. The scaling law for the moments of this measure,

$$
\frac{\left\langle\chi(r)^{p}\right\rangle}{\langle\chi(r)\rangle^{p}} \sim r^{-\mu_{p}},
$$

is an important characteristic of the dissipation intermittency [16, 18]. Using Taylor's hypothesis, one can define the local dissipation rate as

$$
\chi(\tau) \sim \frac{\int_{0}^{\tau}\left(\frac{d T}{d t}\right)^{2} d t}{\tau},
$$

and the corresponding scaling of the moments of the local dissipation rate [18] as

$$
\frac{\left\langle\chi(\tau)^{p}\right\rangle}{\langle\chi(\tau)\rangle^{p}} \sim \tau^{-\mu_{p}} .
$$

Analogous considerations can be brought to bear for the magnitude of the temperature gradient

$$
\chi_{*}(\tau) \sim \frac{\int_{0}^{\tau}\left(\frac{d G}{d t}\right)^{2} d t}{\tau},
$$



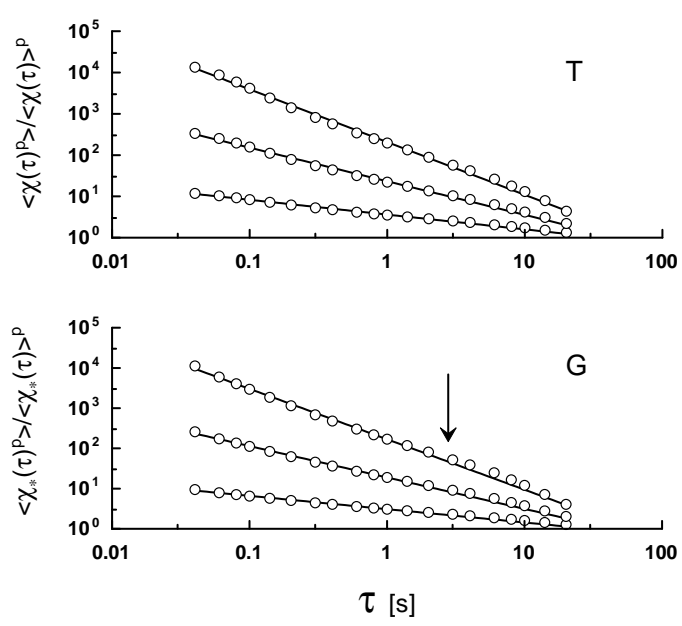

FIG. 3: Normalized moments $\left\langle\chi(\tau)^{p}\right\rangle$ and $\left\langle\chi_{*}(\tau)^{p}\right\rangle$ against $\tau$ for the data obtained in convection $(p=2,3,4)$. The straight lines drawn to indicate scaling are best fits to the data on the left of the vertical arrow.

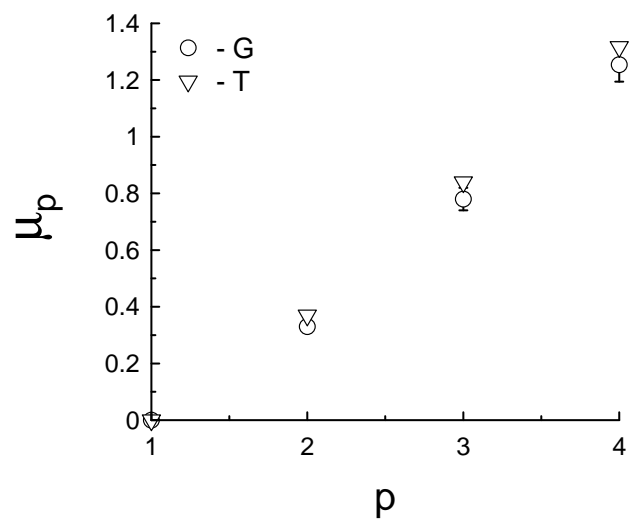

FIG. 4: Intermittency exponents $\mu_{p}$ and $\mu_{p}^{*}$ extracted as slopes of the straight lines in Fig. 3 (equation (18)).

with exponents $\mu_{p}^{*}$. We show in Fig. 3 the dependence of the normalized moments $\left\langle\chi(\tau)^{p}\right\rangle$ and $\left\langle\chi_{*}(\tau)^{p}\right\rangle$ on $\tau$ calculated for the data obtained in thermal convection. The slopes of these straight lines provide us with the values of the intermittency exponents $\mu_{p}$ and $\mu_{p}^{*}$, which are shown in Fig. 4. The two sets of intermittency exponents obtained for $T$ and $G$ are very close.

In summary, we have derived an equation for the magnitude of the temperature gradient $G$ in thermal convection, and shown that there are general circumstances under which the equation is identical to that governing the temperature itself. The main difference is that the velocity appearing the new equation, being a conditional average, is a smoothed field. For the advection-diffusion equation governed by a smooth velocity field, it is natural to expect a power-law spectrum with a slope of -1 ; measurements of the magnitude of the spectral density of $G$ are consistent with this expectation. The correlation function of $G$ shows a logarithmic behavior, also as expected. Finally, the scaling of the square of the derivative of $G$ has scaling exponents that are identical to those of the temperature itself, confirming that a deep analogy exists between $T$ and $G$ in the inertial range.

We thank J. Schumacher and V. Yakhot for brief discussions at an early stage of the work.

[1] B. Castaing, G. Gunaratne, F. Heslot, L. Kadanoff, A. Libchaber, S. Thomae, X.-Z. Wu, A. Zaleski and G. Zanetti, J. Fluid Mech. 204, 1 (1989)

[2] V. Yakhot, Phys. Rev. Lett. 69, 769 (1992)

[3] J.A. Glaizer, T. Segawa, T. Naert and M. Sano, Nature 398, 307 (1999)

[4] S. Ashkenazi and V. Steineberg, Phys. Rev. Lett. 83, 4760 (1999)

[5] Y.-B. Du and P. Tong, J. Fluid Mech. 407, 57 (2000)

[6] S. Grossmann and D. Lohse, J. Fluid Mech. 407, 27 (2000); Phys. Rev. E 66, art. 016305 (2002)

[7] J.J. Niemela, L. Skrbek, K.R. Sreenivasan and R.J. Donnelly, Nature 404, 837 (2000); J.J. Niemela and K.R. Sreenivasan, J. Fluid Mech. 481, 355 (2003)

[8] X. Xu, K.M.S. Bajaj and G. Ahlers, Phys. Rev. Lett. 84, $4357(2000)$

[9] X. Chavanne, F. Chilla, B. Chabaud, B. Castaing and B. Hebral, Phys. Fluids 13, 1300 (2001)

[10] S.-Q. Zhou and K.-Q. Xia, Phys. Rev. Lett. 87, 064501 (2001); K.-Q. Xia, C. Sun and S.-Q. Zhou, Phys. Rev. E 68, 066303 (2003)

[11] R. Verzicco and R. Camussi, J. Fluid Mech. 477, 19 (2003)

[12] E.S.C. Ching, Y. Cohen, T. Gilbert and I. Procaccia, Phys. Rev. E 67, 016304 (2003)

[13] Ya.B. Zeldovich, A.A. Ruzmaikin and D.D. Sokoloff, Magnetic Fields in Astrophysics (Gordon and Breach) 1983; Ya.B. Zeldovich, B. Molchanov, A.A. Ruzmaikin, D.D. Sokolov Sov. Phys. Usp. 30, 353 (1987)

[14] G.-C. Yuan, K. Nam, T.M. Antonsen, Jr., E. Ott, and P.N. Guzdar, Chaos 10, 39 (2000)

[15] G.K. Batchelor, J. Fluid Mech. 5, 113 (1959)

[16] A.S. Monin and A.M. Yaglom, Statistical Fluid Mechanics, Vol. 2, (MIT Press, Cambridge 1975)

[17] M. Chertkov, G. Falkovich, I. Kolokolov and V. Lebedev, Phys. Rev. E 51, 5609 (1995)

[18] K.R. Sreenivasan and R.A. Antonia, Annu. Rev. Fluid Mech. 29, 435 (1997) 